\title{
Legislación argentina sobre polución lumínica
}

Verónica Presa ${ }^{1}$ y Ricardo Picicelli ${ }^{1}$

\section{Resumen}

La utilización de luminarias que iluminan en direcciones $u$ horarios innecesarios es una de las causas fundamentales de la existencia de la polución lumínica. La misma afecta la salud de las personas y a muchas especies animales, y es la que menos se nota o preocupa. Este hecho provoca que casi no exista legislación al respecto, o que la existente sea incompleta. En el presente trabajo, se exhiben, analizan y comparan las legislaciones ambientales en la Argentina y en otros países del mundo.

Palabras clave: polución lumínica, contaminación lumínica, legislación ambiental.

\section{Abstract}

One of the main causes of light pollution is the misuse of illumination devices including propagation in unnecessary directions, intensity or hours. This affects people's health and numerous animal species, and it is the one which is less noticed or paid attention to. As a consequence of this, there is almost no legislation regarding the issue and the current laws are incomplete. In the present work, several countries' -including Argentina's- environmental laws are exhibited, analyzed and compared.

Keywords: light pollution, light contamination, environmental legislation. 


\section{Introducción}

La contaminación o polución lumínica es producto del conjunto de luminarias ambientales, alumbrado público y otros agentes que producen luz en intensidades, direcciones $u$ horarios innecesarios para las actividades que se realizan en el lugar donde están instaladas dichas luminarias, alterando la oscuridad natural del habitad.

Para tener idea de la dimensión de esta problemática, la Organización de las Naciones Unidas para la Educación, la Ciencia y la Cultura (UNESCO) en su Declaración Universal de los Derechos de las Generaciones Futuras afirma que "Las personas de las generaciones futuras tienen el derecho a una Tierra indemne y no contaminada, incluyendo el derecho a un cielo puro".

Por su parte, la Constitución Nacional en su artículo 41 establece que "Todos los habitantes gozan del derecho a un ambiente sano, equilibrado, apto para el desarrollo humano y para que las actividades productivas satisfagan las necesidades presentes sin comprometer las de las generaciones futuras; y tienen el deber de preservarlo.........Las autoridades proveerán a la protección de este derecho, a la utilización racional de los recursos naturales, a la preservación del patrimonio natural y cultural y de la diversidad biológica, y a la información y educación ambiental. Corresponde a la Nación dictar las normas que contengan los presupuestos mínimos de protección, y a las provincias, las necesarias para complementarlas, sin que aquellas alteren las jurisdicciones locales....".

En los centros urbanos la mayoría de los habitantes desconoce en qué medida el exceso de luz afecta su salud y deteriora el ambiente, agravado esto por el hecho que nuestro país posee una escasa legislación atinente a dicha problemática.

Esto contrasta con otros países desarrollados donde en los últimos años se llevó a cabo un esfuerzo legislativo importante para poder controlar este fenómeno e indirectamente concientizar a la población.

La polución lumínica afecta la salud de la población, altera los ciclos vitales de numerosas especies y dificulta la visión del cielo para la observación astronómica. Por otro lado el uso ineficiente de la iluminación implica un exceso de la energía utilizada que agrava los problemas ambientales por la posible generación de dióxido de carbono.

\section{Consecuencias de la Polución Lumínica}

Está científicamente probado que una excesiva exposición a la luz en horarios nocturnos tiene connotaciones negativas sobre la fisiología humana y animal, interfiriendo la producción de melatonina por parte de la glándula pineal. Dicha hormona, que se secreta especialmente durante la noche, tiene efectos positivos sobre la salud como ser la regulación del sueño, efectos antioxidantes 
y anticancerígenos, la sincronización, la regulación de los ritmos circadianos, es reductora de la endotelina-1 (péptido con gran capacidad vasoconstrictora asociada con el desarrollo y crecimiento de células cancerosas), entre otros aspectos positivos.

Cuando se produce una exposición excesiva a la luz durante la noche, en especial longitudes de onda cercanas al azul, la glándula pineal restringe la síntesis de melatonina debido a que la luz es transducida a una señal eléctrica que confluye al sistema nervioso central, alterando la función del reloj biológico.

La polución lumínica genera, de esta manera, cambios fisiológicos que acumulados pueden generar la aparición de diversos trastornos de salud como ser alteraciones en el sueño, ansiedad, aumento de la agresividad, dolores de cabeza, disfunciones sexuales, fatiga visual, insomnio, envejecimiento prematuro y desarrollo de tumores.

Por otra parte, la contaminación lumínica convierte la noche en día en las ciudades y zonas aledañas, con consecuencias ecológicas adversas de alto impacto. Para muchas especies animales su pico de actividad ocurre durante la noche. El exceso de luz implica un cambio en el hábito de muchas especies, incluyendo la desaparición de algunas y generando de esta manera un grave daño en el ecosistema.

La polución lumínica también ocasiona un gran problema para la actividad astronómica, impidiendo la adecuada observación del cielo nocturno hasta el punto de alterar la actividad de ciertos observatorios que, en su momento han sido instalados en zonas alejadas de centros urbanos y/o a grandes alturas en búsqueda de cielos diáfanos huyendo de la polución lumínica, que inexorablemente los alcanzó. La causa de esto es que el fenómeno de la propagación de la luz en la atmósfera hace que sus efectos negativos se hagan evidentes hasta distancias de cientos de kilómetros de las fuentes emisoras. Esto no sólo genera un daño a la investigación científica, sino un fuerte impacto económico negativo ya que torna inutilizables instrumentos que han resultado altamente costosos.

\section{Legislación en la República Argentina}

A pesar del mandato de la Constitución Nacional en su artículo 41 para que la Nación y las Provincias dicten las leyes necesarias para cuidar el cielo nocturno, y de la Ley Nacional № 25675/2002 "Ley General de Ambiente"; la legislación en materia ambiental existente en Argentina se encuentra dispersa en diversas leyes que tratan problemáticas específicas, dada la inexistencia de un Código de Derecho Ambiental, como ocurre en otros países más avanzados en dicha temática.

A partir de la reforma de la Constitución Nacional en 1994, se estableció un régimen por el cual se dispone que la Nación y las Provincias tengan a su cargo el dictado de leyes de protección ambiental. 
Hasta el momento se han sancionado las siguientes leyes nacionales de presupuestos mínimos: la Ley N 25675/2002 "Ley de Política Ambiental Nacional" y la Ley $N^{\circ}$ 25831/2003 "Régimen de Libre Acceso a la Información Pública Ambiental". Estas tocan de forma muy amplia toda la problemática sin hacer hincapié en ninguna forma específica de contaminación.

La ley 25675, en su artículo 1, establece los presupuestos mínimos para el logro de una gestión sustentable y adecuada para el ambiente, la preservación y protección de la diversidad biológica y la implementación del desarrollo sustentable.

Esta ley fija los objetivos a cumplir, como la de preservar, conservar y recuperar los recursos naturales, inculcar en el ciudadano la conciencia por el cuidado del ambiente y el uso racional y sustentable de los recursos naturales, mantener el equilibrio ecológico asegurando la conservación de la diversidad biológica, establecer un sistema federal de coordinación para la implementación de políticas atinentes a la problemática ambiental, entre los más destacados. Está claro que la mayor parte de estos objetivos no son alcanzados por el perjuicio que la polución lumínica genera sobre el ambiente y los seres vivos.

En su artículo 4, prevé que la aplicación e interpretación de dicha ley sea sujeta al cumplimiento de ciertos principios: congruencia, prevención, precautorio, equidad intergeneracional, progresividad, responsabilidad, subsidiariedad, sustentabilidad, solidaridad y cooperación, por los que pasa el nudo conceptual de la ley.

El artículo 11 de evaluación del impacto ambiental, sostiene que toda obra o actividad que altere el ambiente deberá estar sujeta a una previa revisión. ¿Cumplen realmente este artículo las autoridades nacionales, provinciales y municipales, o la inspección se hace a posteriori del daño causado?

Tanto los artículos 14 y 15 de Educación Ambiental así como del 19 al 21 de Participación Ciudadana son clave para permitirle a los individuos inmiscuirse en asuntos ambientales, y así despertar en ellos una conciencia por la preservación del ambiente.

La ley prevé la creación del Sistema Federal Ambiental cuyo objetivo sea la coordinación de políticas ambientales entre el Gobierno Nacional y los Provinciales, el cual será instrumentado por el Consejo Federal de Medio Ambiente (COFEMA).

Por último, en los artículos 27 a 34 la ley dicta una serie de normas atinentes a responsabilizar y penar a todo aquel que genere un daño al ambiente, ya sea reparable (obligándolo a reestablecer el ambiente a su estado inicial), o irreparables (obligándolo a pagar la indemnización que determine la justicia).

Notar, en relación con la problemática central de este trabajo, que la polución lumínica tiene una ventaja por sobre otros tipos de contaminaciones: en general los daños causados por esta son reparables. Sólo basta con dejar de iluminar ineficientemente.

La ley 25831, tiene por objeto establecer los presupuestos mínimos de protección ambiental para garantizar el derecho de acceso a la información que se encontrare 
en poder del Estado, tanto en el ámbito nacional como provincial, municipal y de la Ciudad de Buenos Aires, así como también de entes autárquicos y empresas prestadoras de servicios públicos, sean públicas, privadas o mixtas.

En ella define información ambiental a toda aquella información en cualquier forma de expresión o soporte relacionada con el ambiente, los recursos naturales o culturales y el desarrollo sustentable. Nótese lo amplia y ambigua que es esta definición.

La ley deja sentado que el acceso a la información ambiental será libre y gratuito, salvo ciertos gastos administrativos que devengan de la entrega de los mismos e indica quiénes son los sujetos alcanzados por esta ley.

Más allá de las excepciones a la obligatoriedad de entrega de información, la ley hace referencia a las infracciones a esta ley, como ser obstrucción, falsedad, ocultamiento o falta de respuesta dentro de los límites encuadrados por la misma. Los funcionarios públicos que caigan en esta infracción, serán pasibles de la aplicación de las sanciones previstas en la Ley N 25164/1999 "Ley Marco de Regulación de Empleo Público Nacional”. Las empresas de servicios públicos que no cumplan con las obligaciones exigidas por la ley, serán pasibles de las sanciones previstas en las normas o contratos de la concesión correspondiente, aparte de las responsabilidades civiles y penales pertinentes.

Cabe aclarar que esta ley está subordinada a la ley 25675, en virtud de la supremacía que esta última asumió frente a las demás leyes ambientales federales en sus artículos 3 y 4 . De hecho, los artículos 16, 17 y 18 de la ley 25675 son un compendio de la ley de Información Ambiental.

A pesar de contar con una grandilocuente Ley Nacional de Política Ambiental haciendo referencia a todo tipo de contaminación en general y ninguna en particular, la República Argentina sólo cuenta con un puñado de Leyes Provinciales u Ordenanzas Municipales concretas acerca de la contaminación lumínica. Y no es casual que sea en distritos que tienen instalado un importante centro de observación astronómica, estando detrás de esto principalmente el motivo económico.

La Cámara de Diputados de la Provincia de San Juan sancionó en 1987 la Ley N ${ }^{\circ} 5771$ "Ley de Protección del Cielo" en las inmediaciones del complejo astronómico "El Leoncito", figura 1 izq., preservando dicha área de todos los factores o actividades que generen contaminación del cielo en un radio de $15 \mathrm{~km}$. Esta es la única ley de carácter provincial sancionada en la República Argentina.

La misma prohíbe el uso de artefactos de iluminación artificial no regulados que emitan en el rango de longitudes de onda entre $300 \mathrm{y} 1000 \mathrm{~nm}$, incluyendo las luces utilizadas para la señalización de las rutas.

Anteriormente a esta ley en 1979, mediante el Decreto Ley Provincial de San Juan $\mathrm{N}^{\circ} 4525$, se había creado la Reserva Astronómica en la cual se emplaza dicho observatorio.

A posteriori, mediante el Decreto Nacional № 2148/1990, el Poder Ejecutivo 
Nacional creó la categoría de Reserva Natural Estricta...."que reduce al mínimo posible la interferencia humana directa en las áreas que con esa denominación sean designadas, asegurándose así, que las comunidades naturales incluyendo todas las especies que las integran y los procesos ecológicos se desarrollen en forma natural". Luego, mediante el Decreto Nacional N $46 / 1994$ se crea la Reserva Natural Estricta El Leoncito. Finalmente, la Ley N ${ }^{\circ} 6764 / 1996$ de la Provincia de San Juan cede al Estado Nacional dicha jurisdicción con el objeto que sea declarada Parque Nacional, de acuerdo al régimen establecido por la Ley Nacional N²2351/1980 de Parques Nacionales, Monumentos Naturales y Reservas Nacionales.
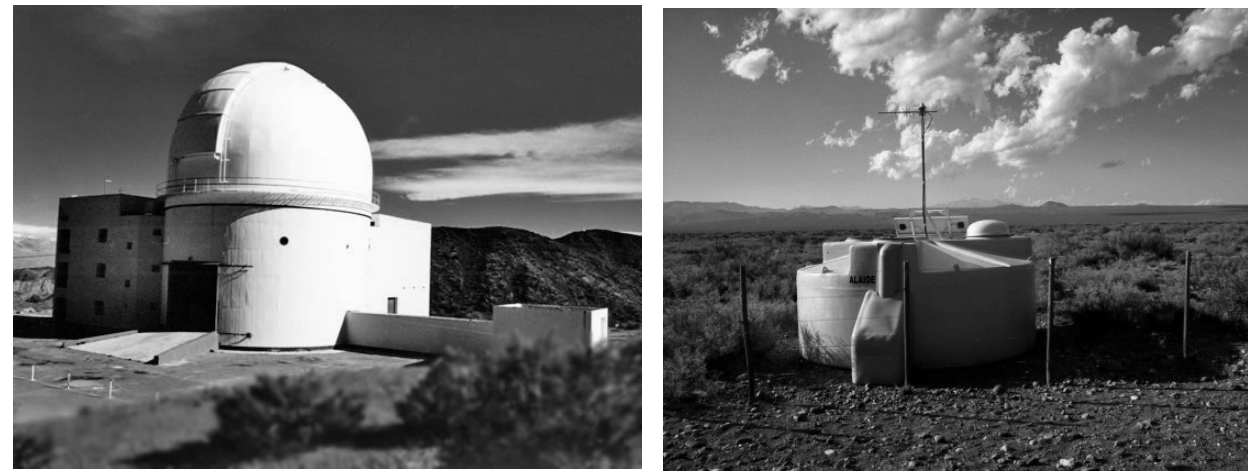

Figura 1: Izq: Observatorio astronómico El Leoncito. (Foto archivo: Travel San Juan). Der: Observatorio Pierre Auger (http://www.fotosimagenes.org/observatorio-pierre-auger).

En el Municipio de Malargüe, Provincia de Mendoza, está instalado el observatorio de rayos cósmicos del Proyecto Pierre Auger, figura 1 derecha. Posee una escueta pero profunda Ordenanza, la $N^{\circ} 1298 / 2005$, inherente a la polución lumínica. En su considerando previo, no sólo hace referencia al impacto negativo que la polución en especial la lumínica, ejerce sobre la actividad de este importante centro científico, sino que recalca los perjuicios que recaen sobre la flora, fauna y en especial sobre los seres humanos. ......"que la sensación de calma y bienestar que normalmente genera observar el cielo estrellado es algo necesario, teniendo en cuenta la velocidad con la que se desarrolla la vida moderna del ser humano y el stress que esto produce". Inclusive remarca el hecho de conservar el cielo por una cuestión meramente turística, para fortalecer las actividades de Turismo Aventura y Turismo Ecológico.

En su artículo 2 ordena......”mantener al máximo posible las condiciones naturales de las horas nocturnas, en beneficio de la flora, fauna y los ecosistemas en general",....."promover la ecoeficiencia mediante el ahorro de energía.......sin que esto perjudique la seguridad de las personas",....” evitar la intrusión lumínica del entorno doméstico minimizando las molestias y/o perjuicios", ,..." prevenir y corregir los efectos de la contaminación lumínica sobre la visión del cielo". 
El artículo 4 hace mención a convenios entre la Municipalidad, el Observatorio Pierre Auger, Universidades, Empresas de distribución eléctrica y Entes Reguladores. De esta manera logra una articulación entre los distintos sectores involucrados.

Si bien la ordenanza no hace demasiada referencia a cuestiones técnicas para prevenir y/o corregir los efectos de la polución sobre el cielo nocturno y la forma que afecta científica y económicamente al Observatorio, sí se refiere con especial cuidado a los efectos adversos sobre los seres vivos.

La Municipalidad de Rosario, Provincia de Santa Fe, sancionó en 2000 la Ordenanza $\mathrm{N}^{\circ} 6939$ en la cual establece un régimen de alumbrado público en toda la ciudad de Rosario para proteger al cielo de la contaminación luminosa.

En la misma presenta una completa definición de polución lumínica para no dar lugar a interpretaciones ambiguas y confusas. Asimismo, da una minuciosa descripción de instalación de alumbrado público que quedará dentro del marco de la Ordenanza: alumbrado vial y ornamental, letreros, anuncios luminosos y vidrieras comerciales, alumbrado de establecimientos recreativos y deportivos, alumbrado de seguridad y alumbrado de viviendas particulares y edificios públicos.

En su artículo 11 expresa...."previo al otorgamiento de toda habilitación para instalación de letreros, carteles y anuncios publicitarios, será indispensable comprobar que la instalación luminosa proyectada cumpla con lo establecido en la presente Ordenanza y su reglamentación"

En cuanto al alumbrado público, incita a evitar el uso de lámparas de vapor de mercurio,...."privilegiando el uso de luminarias de vapor de sodio $u$ otras que eviten un elevado consumo energético". Nótese que para la fecha en que fue promulgada dicha Ordenanza, todavía no estaba difundido el uso de tecnología led.

En el artículo 13, hace una importante acotación a las luminarias tipo globo, una de las principales fuentes de polución procedente del alumbrado público; permitiendo sólo las que no generen pérdidas a través de su hemisferio superior, el cual deberá estar tapado, alunizado interiormente o pintado de negro.

En dicha Ordenanza se hace referencia al uso de proyectores y/o laser con fines recreativos o publicitarios, dejando expresamente prohibido su uso en caso de interferir con observaciones astronómicas. Sin embargo, en la misma no se genera ninguna discusión acerca de los efectos negativos sobre los seres vivos, en especial sobre los seres humanos.

\section{Legislación en otros países}

La comunidad internacional, en especial Europa, países del Norte de América y Australia, son los más preocupados y los que llevan la delantera en leyes que regulen la polución lumínica en sus estados. Sin embargo la gran mayoría son cualitativas 
y no dan los detalles para su instrumentación y puesta en práctica.

En Estados Unidos, existen leyes estatales en la mayoría de sus estados, las cuales han sido propuestas por asociaciones como la Internacional Dark Sky Asociación y la Illumitating Engineering Society of North America.

España es, sin duda alguna, el país que ha despertado mayor concientización de la problemática, en donde se encuentra un gran volumen de publicaciones al respecto y más ha legislado en sus regiones autónomas. En particular, las Islas Canarias prevé desde 1988 la regulación de la polución lumínica ya que en dichas islas está instalado el Observatorio de Astrofísica de Canarias. En Cataluña existe una normativa muy específica desde 2001 en cuanto al tipo de lámparas, luminarias, encendido de las mismas por reloj astronómico y reducción de potencia.

En el resto de los países europeos, el Reino Unido desarrolla un plan a 15 años (2001-2015) para reducir la polución lumínica en forma gradual; lo mismo ocurre en Alemania, e Italia cubre las dos terceras partes de su territorio con leyes destinadas a la protección del cielo. Cabe destacar la normativa de Eslovenia de 2007 que es la más restrictiva con respecto a que la emisión por encima del horizonte sea del $0 \%$. Dicha reglamentación, establecida por el gobierno, nunca ha sido votada en el parlamento y puede ser modificada en cualquier momento, como ocurrió en 2010 para la inauguración del Stadion Stožice. La FIFA había amenazado con no homologar el estadio si no respetaba las referencias lumínicas impuestas, algo imposible de cumplir acorde a dicho estatuto. El gobierno terminó modificándola para autorizar las lámparas cuya emisión de flujo luminoso hacia el cielo fuera de $0,5 \%$, la cifra más baja del mundo para este tipo de eventos.

En Francia, desde Julio de 2013 entró en vigencia una normativa para el apagado de luces entre la una y las siete de la mañana, y en los próximos dos años también se extenderá a la iluminación de carteles publicitarios. Este plan no sólo se instrumenta en Paris, sino también en todas las ciudades de Francia. Si bien las medidas apuntan principalmente a ahorrar energía y dinero, el gobierno también tiene la intención de reducir el impacto que la iluminación artificial provoca en el ambiente nocturno, consecuencias que recientemente se están empezando a tener en cuenta. Sin embargo, las medidas han generado protestas entre los comerciantes de Paris, más preocupados por seguir siendo uno de los destinos turísticos más importantes del mundo y por preservar la legendaria fama de su ciudad de seguir llamándose "La Ciudad Luz". Figura 2. 

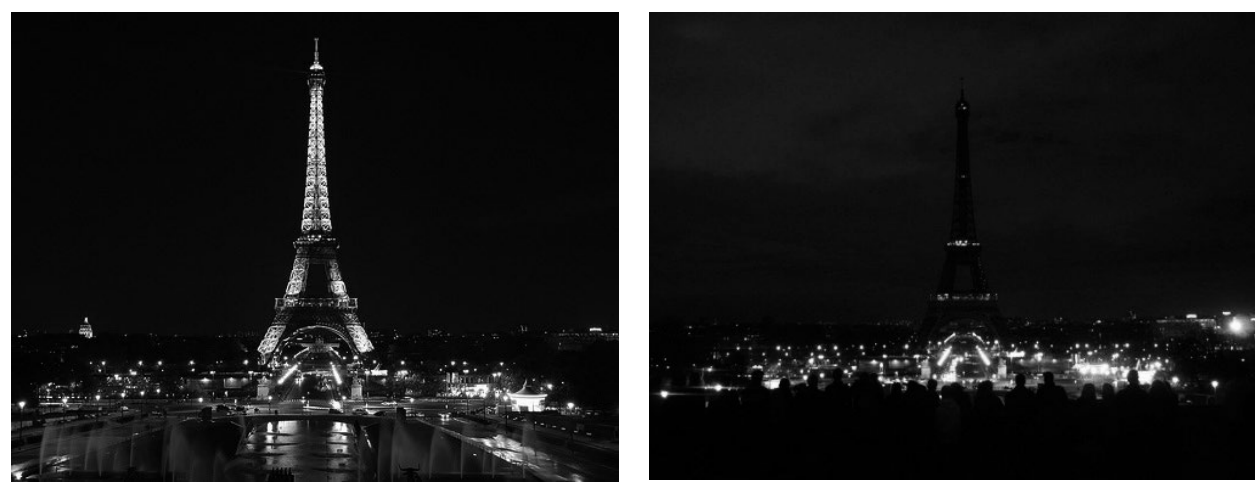

Figura 2: Torre Eiffel antes y después de la nueva normativa

Los sectores de la superficie terrestre menos contaminados desde el punto de vista lumínico son: América del Sur, junto con el centro de Australia, Sahara y Siberia. Figura 3.

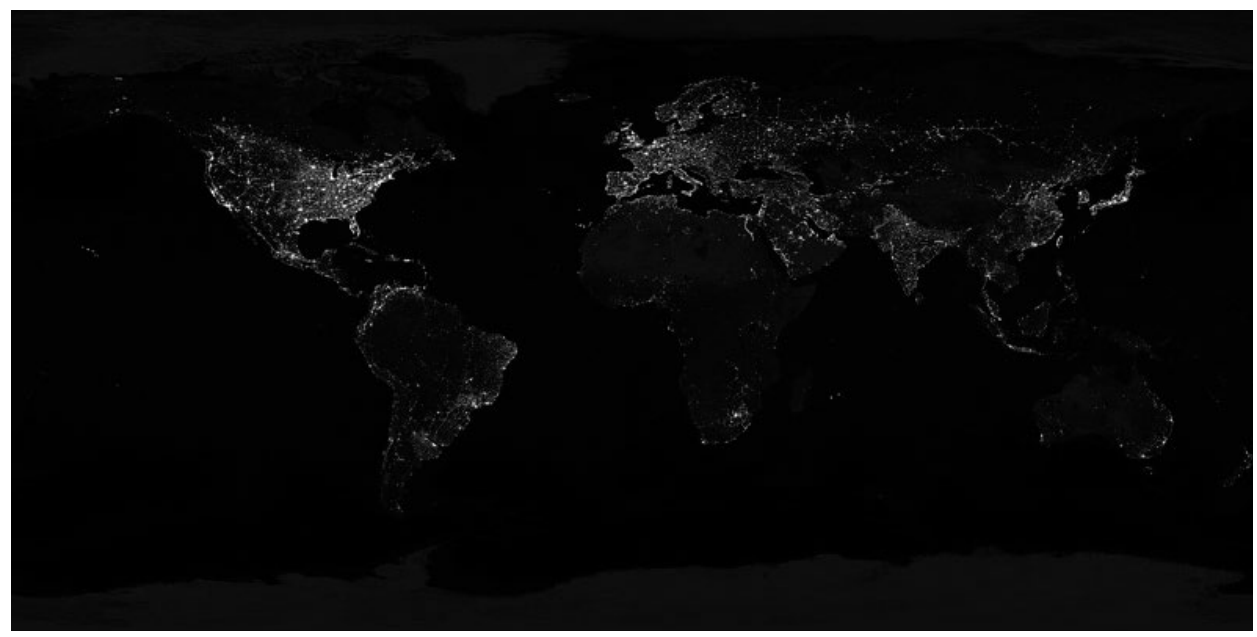

Figura 3: La Tierra de Noche, tomada por diversos satélites meteorológicos del ejército estadounidense 2006 (Foto: NASA)

Chile posee legislación desde 1995 con las leyes N 19300 sobre "Bases Generales del Medio Ambiente" y la ley No 18410 de "Creación de la Superintendencia de Electricidad y Combustible". La concientización del problema hizo que en 2012 se establezca una modernización y adaptación a los nuevos requerimientos dictando normas de calidad ambiental y regulación de la contaminación lumínica. Lamentablemente, como ocurre en nuestro país, en Canarias y en Chile, la normativa fuerte y específica se asocia con la calidad astronómica del cielo y a la presencia de observatorios y no por los impactos ecológicos que rompen los patrones naturales que afectan a muchos seres vivos incluyendo a la raza humana. 


\section{Conclusiones}

Con el devenir de la Revolución Industrial, se produjo un repentino cambio tecnológico que generó un gran desarrollo productivo originado en los principales países europeos, extendida a posteriori al resto del mundo. Esto generó un proceso acelerado de cambio y destrucción del ambiente que en los últimos años ha comenzado a instalarse en la agenda mundial. La mayoría de los países cuenta con legislación al respecto e incluso se mencionan en sus Cartas Magnas, como es el caso de la República Argentina.

Nuestra Constitución, con la reforma de 1994, consagra el derecho humano al medio ambiente en su artículo 41, lo cual denota que el derecho al medio ambiente está inexorablemente ligado a los Derechos Humanos, como un Derecho Fundamental.

Sin embargo, queda mucho por hacer y legislar en materia de Polución Lumínica. Los gobiernos, que son los responsables de regular y multar, no siempre cumplen y hacen cumplir las escasas leyes existentes.

La poca atención destinada a los problemas de polución lumínica puede estar asociada a dos factores. Por un lado al hecho que dentro de todo el espectro de contaminaciones, la lumínica es la menos dañina y la que más fácilmente se puede solucionar; y por otro lado, al escaso conocimiento de la población en cuestiones ambientales, en especial la lumínica. Para revertirla, sólo basta con iluminar racional y eficientemente. Así se lograría un importante ahorro energético, la consecuente disminución de emisiones de $\mathrm{CO} 2$ a la atmósfera y el cuidado, de los ecosistemas y de la salud humana.

En lo que a la República Argentina se refiere, la escasa legislación referente a polución lumínica sólo se circunscribe a regiones que poseen algún importante centro de observación astronómica, pero no se pone énfasis en los severos daños que se generan sobre la flora, fauna y en especial sobre la salud de los seres humanos.

Cuando los habitantes del planeta se concienticen que forman parte del medio ambiente y que sus acciones lo dañan, los problemas de contaminación se irán atenuando hasta encontrar un justo equilibrio entre el desarrollo tecnológico y su hábitat. 


\section{Referenicas}

Baker J. (1990). Toad aggregations under street lamps. British Herpetological Society Bulletin 31:26-27.

Brainard GC, Hanifin JP, Rollag MD, Greeson J, Byrne B, Glickman G, Gener E, Sanford B, (2001). Human melatonin regulation is not mediated by the three cone photopic visual system. J. Clin Endocrinol Mettab. 86:433-436.

Constitución Nacional de la República Argentina, Art. 41, 1994.

Davis S, Mirick DK, Stevens RG. (2001). Night shift work, light at night and risk of breast cancer. J.C. Cancer Institute. 93:1557-1562.

Declaración Universal de los Derechos de las Generaciones Futuras, UNESCO.

Decreto Ley Provincial de San Juan 4525/1979.

Decreto Nacional N²148/1990.

Decreto Nacional N $46 / 1994$.

Documento Final del Grupo de Trabajo GT-Luz "Contaminación Lumínica". (2008). $9^{\circ}$ Congreso Nacional del Medio Ambiente. Cumbre del Desarrollo Sustentable.

Herbert AD, (1970). Spatial disorientation in birds. Wilson Bulletin 82:400-419.

http://www.darksky.org (2013, 21 de marzo).

http://www.excelsior.com.mx/nacional/2013/06/22/905344 (2013, 21 de marzo).

http://www.iac.es/adjuntos/otpc/regcielo.pdf (2013, 21 de marzo).

http://www.ies.org/ (2013, 21 de marzo).

http://www.legislacion-oficial.vlex.cl/vid/norma-nica-elaborada-partir-naofomento-434516086 (2013, 21 de marzo).

http://www.taringa.net/posts/ecologia/11775075/Eslovenia-y-su-ley-a-favor-delas-estrellas-Hagamozlo.html (2013, 21 de marzo).

Instituto de Derecho Ambiental. Directora: Dra. González Gabriela. Comentario de la Ley N 25831 por Mario F. Valls. Disponible en: http://www.caq.org.ar/ despliegue-articulos.php?idarticulo=32 $(2013,21$ de marzo).

Lewy AJ, Wehr TA, Goodwin FK, Newsome DA, Markey SP, (1981). Light 
suppresses melatonin secretion in humans. Science 210, 1267-1269.

Ley de protección del cielo 5771/1987 de la Provincia de San Juan.

Ley N 25164/1999 “Ley Marco de Regulación de Empleo Público Nacional”.

Ley N²5675/2002 de "Política Ambiental Nacional".

Ley $\mathrm{N}^{\circ}$ 25831/2003 "Régimen de Libre Acceso a la Información Pública Ambiental".

Ley N 6764/1996 de la Provincia de San Juan.

Ley Nacional $N^{\circ} 22351 / 1980$ de Parques Nacionales, Monumentos Naturales y Reservas Nacionales.

Longcore T, Rich C, (2004). Ecological light pollution. Frontiers in Ecology and the Environment 2(4), 191-198.

Nikolaus G, Pearson DJ, (1983). Attraction of nocturnal migrants to car headlights in the Chyulu Hills, Kenya. Scopus 4:45-46.

Ordenanza N 1298/2005 de la Municipalidad de Malargüe, Provincia de Mendoza.

Ordenanza N 6939/2000 de la Municipalidad de Rosario, Provincia de Santa Fe.

Petteri Teikari, (2007). Light Pollution: Definition, legislation, measurement, modeling and environmental effects. Universidad Politécnica de Catalunya, Barcelona, Catalunya.

Schwartz HE (2003). Light Pollution Control: Word-Wide Effects of Efforts to Reduce Light Pollution. Heck A (ed). Organizations and Strategies in Astronomy, Kluwer Academic Publishers. 4, 37-57.

Stevens RG, Davis S, Thomas DB, Anderson LE, Wilson BW. (1992). Electric power, pineal function and the risk of breast cancer. FASEB J 6, 853-860.

Vanecek J. (1998). Cellular mechanisms of melatonin action. Physiol Rev. 78(3), 687-721. 